\title{
MicroRNA-616 promotes the migration, invasion and epithelial-mesenchymal transition of HCC by targeting PTEN
}

\author{
DI ZHANG ${ }^{1}$, PEIHUA ZHOU ${ }^{2}$, WEI WANG ${ }^{2}$, XIAOLONG WANG ${ }^{2}$, JUNHUI LI $^{1}$, XUEJUN SUN ${ }^{2}$ and LI ZHANG ${ }^{1}$ \\ ${ }^{1}$ Department of General Surgery, The Second Affiliated Hospital of Medical College of Xi'an Jiaotong University; \\ ${ }^{2}$ Department of General Surgery, The First Affiliated Hospital of Medical College \\ of Xi'an Jiaotong University, Xi'an, Shaanxi 710004, P.R. China
}

Received July 17, 2015; Accepted September 2, 2015

DOI: $10.3892 / o r .2015 .4334$

\begin{abstract}
MicroRNAs, which can post-transcriptionally regulate gene expression by binding to the 3'-untranslated regions of the mRNAs, have been found to be the critical regulators of the development and progression of hepatocellular carcinoma (HCC). The present study demonstrated for the first time that microRNA-616 (miR-616) was markedly upregulated in HCC tissues, and was associated with the recurrence and metastasis of HCC. Elevated level of miR-616 was correlated with adverse clinicopathological features and poor prognosis of HCC patients. Gain- and loss-of-function studies revealed that miR-616 could potentiate the migration, invasion and the epithelial-mesenchymal transtion (EMT) phenotype of HCC cells. Phosphatase and tensin homolog (PTEN), the predicted target of miR-616 by bioinformatics analysis, was confirmed as a direct downstream target of miR-616 through western blotting, luciferase reporter and immunohistochemical assays. Furthermore, we demonstrated that miR-616 exerted the promoting effects on EMT and metastatic ability of HCC cells through suppressing PTEN expression. Based on these results, we conclude that miR-616 is a promising prognostic biomarker of HCC and targeting miR-616 may be a potential option to prevent the progression of HCC.
\end{abstract}

\section{Introduction}

Hepatocellular carcinoma (HCC) is one of the most common human cancers worldwide and ranks third in cancer-related death (1). Although remarkable achievements have been made (2), the long-term prognosis of HCC patients remains poor, particularly for those in advanced stages, with the 5-year survival rate lower than 5\% (3). Local and systemic metastasis

Correspondence to: Dr Li Zhang, Department of General Surgery, The Second Affiliated Hospital of Xi'an Jiaotong University, 157 Xiwu Road, Xi'an, Shaanxi 710004, P.R. China

E-mail: lizhang15717@163.com

Key words: microRNA-616, hepatocellular carcinoma, cancer metastasis, epithelial-mesenchymal transition, phosphatase and tensin homolog are the critical reasons for the unsatisfactory survival of HCC patients in advanced stages (4). Elucidating the molecular mechanism of the metastasis of HCC is critical for identifying novel therapeutic targets and may significantly improve the prognosis of HCC patients.

MicroRNAs (miRNAs) are a group of short non-conding RNAs and serve as important post-transcriptional modulator of gene expression by binding to 3' untranslated region (3'-UTR) of targeted mRNAs and resulting in the suppression of protein translation or the degradation of the target mRNAs $(5,6)$. Numerous studies have demonstrated that miRNAs are involved in various cellular activities (7-9) including proliferation, apoptosis, differentiation and movement. Aberrant expression and function of miRNAs has been found to play fundamental roles in human malignancies (7,10-12). In addition, miRNAs have become promising diagnostic and prognostic biomarkers and attractive therapeutic targets of human cancers $(13,14)$.

Among numerous miRNAs, microRNA-616 (miR-616) has been found to be a novel cancer-associated miRNA. miRNA profiling of human gastric cancer showed that miR-616 was overexpressed in gastric cancer tissues (15). In addition, studies of lung (16) and prostate cancer (17) demonstrated that the level of miR-616 was significantly elevated in the serum of patients. Furthermore, miR-616 was found to play an important role in the development and maintenance of androgen-independent prostate cancer by regulating the expression of TFPI-2 (17). However, the expression and clinical significance of miR-616 in HCC tissues, and its functional role and the underlying mechanisms in HCC cells, are still undefined.

In the present study, we demonstrated that miR-616 was significantly upregulated in HCC tissues. Elevated expression of miR-616 was observed in patients with metastasis and recurrence. Additionally, elevated miR-616 expression was associated with adverse clinicopathological features and poor prognosis of HCC patients. In vitro studies showed that miR-616 promoted the invasive behavior and epithelial-mesenchymal transition (EMT) of HCC cells. Moreover, phosphatase and tensin homolog (PTEN) was identified to be the direct downstream target of miR-616 in HCC. miR-616 contributed to the metastasis and EMT of HCC by inhibiting the expression of PTEN. 


\section{Materials and methods}

Human tissue specimens and cell lines. The paired HCC and adjacent non-tumor tissues were collected from $80 \mathrm{HCC}$ patients who received surgical resection of primary HCC in the Department of General Surgery at the Second Affiliated Hospital of Xi'an Jiaotong University from January 2006 to December 2009. No local or systemic treatments had been performed before the surgical resection. Informed consent was obtained from each patient before collecting the clinical samples. The demographic and clinicopathological data are presented in Table I. All protocols in the present study were approved by the Xi'an Jiaotong University Ethics Committee according to the Declaration of Helsinki (as revised in Tokyo, 2004).

Six HCC cell lines, Hep3B, HepG2, Huh7, MHCC97L HCCLM3 and MHCC97H, and the human immortalized normal hepatocyte cell line, LO2 (The Institute of Biochemistry and Cell Biology, Chinese Academy of Sciences, Shanghai, China) were maintained in complete Dulbecco's modified Eagle's medium (DMEM; HyClone, Logan, UT, USA) supplemented with $10 \%$ fetal bovine serum (FBS, HyClone, Thermo Fisher Scientific, Australia) with $100 \mathrm{U} / \mathrm{ml}$ penicillin and $100 \mu \mathrm{g} / \mathrm{ml}$ streptomycin (Sigma, USA) in a humidified incubator containing of $5 \% \mathrm{CO}_{2}$ at $37^{\circ} \mathrm{C}$.

Quantitative real-time PCR ( $q P C R$ ) analysis. Total RNA from tissues or cells was extracted using TRIzol (Life Technologies) according to the manufacturer's instructions. The PCR amplification for the quantification of the miR-616 was performed using the TaqMan microRNA assay kit (Applied Biosystems, Foster City, CA, USA). U6 was used as endogenous controls. The relative expression of miR-616 was normalized to that of U6.

Cell transfection. miR-616 expression vector (HmiR0186MR04), the control vector for miR-616 (CmiR0001-MR04), miR-616 inhibitor (HmiR-AN0721-AM03) and the negative control for the miR-616 inhibitor (CmiR-AN0001-AM03), were purchased from GeneCopoeia (Guangzhou, China). Plasmids carrying wt SMAD7 (pCMV5-SMAD7) were obtained from Addgene (Cambridge, MA, USA). The vectors mentioned above were transfected into the HCC cells using Lipofectamine 2000 (Invitrogen, USA) following the manufacturer's protocol.

Migration and invasion assays. Cell migration and invasion assays were evaluated in chambers of $8-\mu \mathrm{m}$ with Transwell inserts (Millipore, USA) according to the manufacturer's instructions. Cells $\left(5 \times 10^{4}\right)$ were seeded into the top chamber of each insert in serum-free medium and serum-containing medium was used in the lower chamber as the attractant. Specifically for the invasion assay, before seeding the HCC cells, each chamber was coated with mixture of DMEM and Matrigel (Becton-Dickinson Labware, USA) at a ratio of 6:1. After incubated at $37^{\circ} \mathrm{C}$ for $24 \mathrm{~h}$, the cells adherent to the upper surface of the filter were removed using a cotton swab. The migrated or invaded cells were fixed and stained with $0.1 \%$ crystal violet, air-dried and photographed. Three independent experiments were performed.
Table I. The association between the clinicopathological features and miR-616 expression in HCC.

\begin{tabular}{|c|c|c|c|c|}
\hline \multirow{2}{*}{$\begin{array}{l}\text { Clinico- } \\
\text { pathological } \\
\text { features }\end{array}$} & \multirow{2}{*}{$\begin{array}{c}\text { Total no. } \\
\text { of pts. }\end{array}$} & \multicolumn{2}{|c|}{ No. of patients } & \multirow[b]{2}{*}{ P-value } \\
\hline & & $\operatorname{miR}-616^{\mathrm{High}}$ & miR-616 ${ }^{\text {Low }}$ & \\
\hline \multicolumn{5}{|l|}{ Gender } \\
\hline Male & 59 & 31 & 28 & 0.446 \\
\hline Female & 21 & 9 & 12 & \\
\hline \multicolumn{5}{|l|}{ Age (years) } \\
\hline$<50$ & 34 & 18 & 16 & 0.651 \\
\hline$\geq 50$ & 46 & 22 & 24 & \\
\hline \multicolumn{5}{|c|}{ HBV infection } \\
\hline Absent & 28 & 15 & 13 & 0.639 \\
\hline Present & 52 & 25 & 27 & \\
\hline \multicolumn{5}{|l|}{ Cirrhosis } \\
\hline Absent & 33 & 18 & 15 & 0.496 \\
\hline Present & 47 & 22 & 25 & \\
\hline \multicolumn{5}{|l|}{$\begin{array}{l}\text { Diameter of } \\
\text { tumor }(\mathrm{cm})\end{array}$} \\
\hline$<5$ & 34 & 19 & 15 & 0.366 \\
\hline$\geq 5$ & 46 & 21 & 25 & \\
\hline \multicolumn{5}{|c|}{ No. of tumor nodules } \\
\hline 1 & 44 & 24 & 20 & 0.369 \\
\hline$>1$ & 36 & 16 & 20 & \\
\hline \multicolumn{5}{|c|}{ Venous infiltration } \\
\hline Absent & 57 & 24 & 33 & $0.026^{\mathrm{a}}$ \\
\hline Present & 23 & 16 & 7 & \\
\hline \multicolumn{5}{|c|}{$\begin{array}{l}\text { Edmondson-Steiner } \\
\text { grading }\end{array}$} \\
\hline $\mathrm{I}+\mathrm{II}$ & 60 & 25 & 35 & $0.001^{\mathrm{a}}$ \\
\hline III+IV & 20 & 15 & 5 & \\
\hline \multicolumn{5}{|l|}{ TNM stage } \\
\hline $\mathrm{I}+\mathrm{II}$ & 58 & 22 & 36 & $<0.001^{\mathrm{a}}$ \\
\hline III+IV & 22 & 18 & 4 & \\
\hline
\end{tabular}

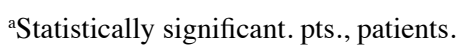

Western blotting. HCC cells were washed twice with phosphate-buffered saline (PBS) and lysed on the culture dishes using RIPA lysis buffer (BioMed, China). Protein concentration was measured using the BCA kit (Pierce, USA) and $30 \mu \mathrm{g}$ of each sample were separated by sodium dodecyl sulfate-polyacrylamide gel electrophoresis and transferred to PVDF membrane. Non-specific binding sites were blocked by incubating with TBST containing 5\% skimmed milk/TBST for $2 \mathrm{~h}$ at room temperature. The blots were then incubated overnight at $4{ }^{\circ} \mathrm{C}$ with the following primary antibodies: PTEN $(1: 1,000)$, AKT $(1: 1,000)$ (both from Santa Cruz, USA), p-AKT $(1: 1,000)$, E-cadherin $(1: 1,000)$, vimentin $(1: 1,000)$ and $\beta$-actin $(1: 1500)$ (all from Cell Signaling, USA). After washing the membranes with TBST, blots were incubated with horseradish peroxidase-conjugated goat anti-mouse or anti-rabbit secondary 

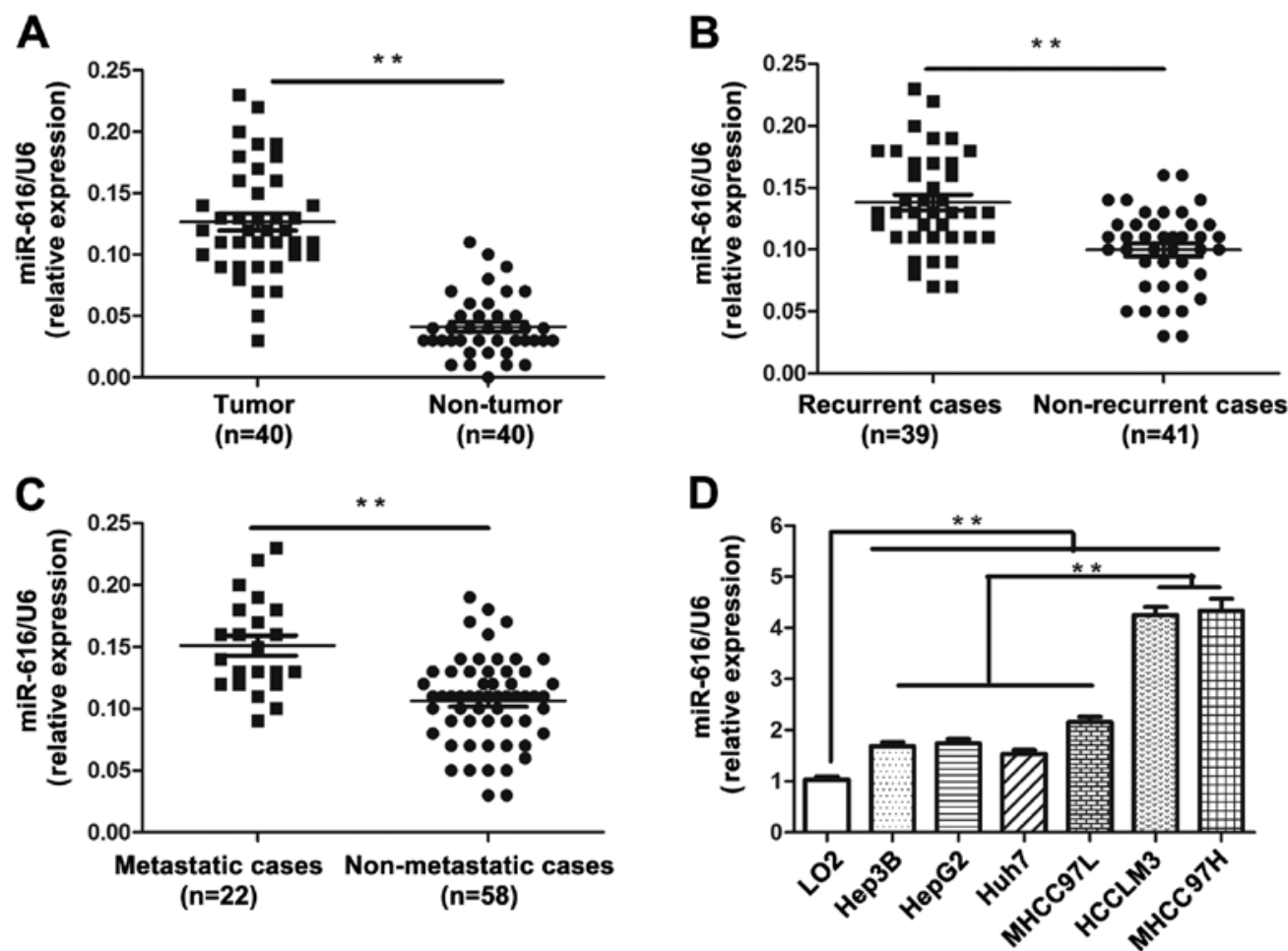

Figure 1. Expression of miR-616 in HCC tissues and cells. Comparing the differences in the expression levels of miR-616 between (A) HCC and adjacent non-tumor tissues, (B) patients with recurrence and those without recurrence, (C) patients with metastases and those without metastases, and (D) HCC cell lines with different metastatic potentials and the immortalized hepatic cell line LO2. ${ }^{* *} \mathrm{P}<0.01$.

antibodies (1:10,000; Bio-Rad, USA), and were detected using the Bio-Rad Gel imaging system.

Luciferase reporter assay. The wild-type 3'-UTR sequence of PTEN (wt PTEN-3'-UTR) predicted to interact with miR-616 or the mutated sequence (mt PTEN-3'-UTR) within the predicted target sites was synthesized and inserted into the pGL3 control vector (Promega, USA). For the reporter assay, Hep3B cells were seeded into 24-well plates and were transfected with the above constructs and miR-616 expressing vector, miR-616 inhibitor, control vector or negative control. After $48 \mathrm{~h}$, the cells were harvested and luciferase activity was measured using the Dual-Luciferase Reporter Assay system (Promega) according to the manufacturer's instructions. Results were obtained from three independent experiments performed in duplicate.

Immunohistochemical staining. After being deparaffinized in xylene and rehydrated in a graded alcohol series and distilled water, the paraffin sections were heated for antigen retrieval in sodium citrate buffer for $10 \mathrm{~min}$, and were quenched for endogenous peroxidase activity in $3 \%$ hydrogen peroxide for $15 \mathrm{~min}$. They were incubated with PTEN (1:100) or p-AKT (1:50) antibody at $4^{\circ} \mathrm{C}$ overnight. Then, sections were incubated with biotinylated secondary antibodies from Zhongshan Golden Bridge Biotechnology (Beijing, China) for $2 \mathrm{~h}$. Sections were stained with the avidin-biotin-peroxidase complex (SABC) method and visualized with diaminobenzidine and counterstained with hematoxylin.

The staining results for PTEN and p-AKT were semiquantitatively calculated by multiplying the staining intensity and the percentage of positive staining. Staining intensity was assessed as four grades: 0 , none; 1 , weak; 2 , moderate; and 3 , strong. The percentage of positive staining was assessed as the following grades: $0,<5 \% ; 1,6-25 \% ; 2,26-50 \% ; 3,51-75 \%$; and $4,>75 \%$. Ten independent high magnification ( $\mathrm{x} 400)$ fields were assayed for each section. Two experienced pathologists independently evaluated the sections, and obtained the average scores of 10 fields.

Statistical analysis. Results are presented as mean \pm SEM. The SPSS statistical package for Windows version 13 (SPSS, Inc., Chicago, IL, USA) and GraphPad Prism 5 software (GraphPad Software, Inc., USA) were employed to perform the statistical analysis. The Pearson's Chi-square test, the Spearman's rank correlation coefficient, the two-tailed Student's t-test, the Kaplan-Meier plot, the log-rank test or ANOVA was used in appropriate situation. Difference was considered to indicate a statistically significant result, at $\mathrm{P}<0.05$.

\section{Results}

miR-616 is upregulated in HCC and is associated with the metastasis and recurrence of HCC. To determine the level of miR-616 expression in HCC tissues, the quantitative RT-PCR assay was performed in 40 pairs of HCC and adjacent nontumor tissues. The expression level of miR-616 in HCC tissues was significantly elevated compared with that in the adjacent non-tumor liver tissues $(\mathrm{P}<0.01$, Fig. $1 \mathrm{~A})$. Moreover, in the recurrent cases, the level of miR-616 was significantly higher than that in the non-recurrent cases $(\mathrm{P}<0.01$, Fig. 1B). In addition, patients with metastasis had obviously increased 
Table II. Multivariate Cox regression analysis of 5-year overall and disease-free survival.

\begin{tabular}{|c|c|c|c|c|c|c|}
\hline \multirow[b]{2}{*}{ Variables } & \multicolumn{3}{|c|}{ Overall survival } & \multicolumn{3}{|c|}{ Disease-free survival } \\
\hline & HR & $95 \% \mathrm{CI}$ & P-value & HR & $95 \% \mathrm{CI}$ & P-value \\
\hline miR-616 & 1.808 & $1.044-3.134$ & $\mathbf{0 . 0 3 5} 5^{\mathrm{a}}$ & 1.724 & $1.014-2.932$ & $0.044^{\mathrm{a}}$ \\
\hline Venous infiltration & 1.941 & $1.012-3.725$ & $0.046^{\mathrm{a}}$ & 2.164 & $1.132-4.139$ & $0.020^{\mathrm{a}}$ \\
\hline Edmondson-Steiner grading & 1.717 & $1.018-2.897$ & $\mathbf{0 . 0 4 3}^{\mathrm{a}}$ & 1.730 & $1.019-2.939$ & $0.042^{\mathrm{a}}$ \\
\hline TNM stage & 2.294 & $1.126-4.671$ & $0.022^{\mathrm{a}}$ & 2.116 & $1.040-4.303$ & $0.038^{\mathrm{a}}$ \\
\hline
\end{tabular}

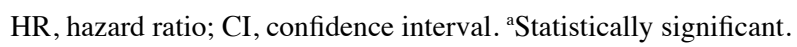

level of miR-616 than those without metastasis $(\mathrm{P}<0.01$, Fig. 1C). These results demonstrated that the expression level of miR-616 is significantly upregulated in HCC tissues and is associated with the recurrence and metastasis of HCC.

Then, we examined the expression level of miR-616 in a non-transformed hepatic cell line (LO2) and six types of HCC cell lines (Hep3B, HepG2, Huh7, MHCC97L, HCCLM3 and MHCC97H). Compared with LO2, all HCC cell lines had significantly higher expression level of miR-616 $(\mathrm{P}<0.01$, Fig. 1D). Moreover, it was interesting to find that miR-616 expression in HCCLM3 and MHCC97H, two highly metastatic HCC cell lines, was obviously higher than that in the low metastatic HCC cell lines including Hep3B, HepG2, Huh7 and MHCC97L $(\mathrm{P}<0.01$, Fig. 1D). These data indicated that miR-616 can probably potentiate the invasive ability of HCC cells.

Elevated expression of miR-616 was correlated with adverse clinicopathological features and poor prognosis of HCC patients. The obvious elevation of miR-616 in HCC tissues and its association with $\mathrm{HCC}$ recurrence and metastasis prompted us to examine the clinical significance of miR-616 in HCC. The expression level of miR-616 was assessed either low $(n=40)$ or high $(n=40)$ based on the cut-off value which was defined as the median level of miR-616 in the 80 patients cohort. As presented in Table II, elevated expression level of miR-616 in HCC patient was associated with the venous infiltration $(\mathrm{P}=0.026)$, high Edmondson-Steiner grading $(\mathrm{P}=0.001)$ and advanced TNM stage $(\mathrm{P}<0.001)$. Furthermore, Kaplan-Meier analysis was performed to evaluate the prognostic value of miR-616 in HCC. Patients with high-miR-616 expression level had significantly decreased overall survival $(\mathrm{P}<0.01$, Fig. 2A) and disease-free survival ( $\mathrm{P}<0.01$, Fig. 2B). These data suggest miR-616 can serve as a prognostic predictor for HCC patients.

miR-616 promotes the migration, invasion and EMT of HCC cells. Next, we further explored the functional role of miR-616 in HCC cells. We transfected Hep3B cells with miR-616 mimics, and miR-616 expression level was significantly increased after transfection, as suggested by the qRT-PCR $(\mathrm{P}<0.01$, Fig. 3A). Functionally, as suggested by the Transwell assay, Hep3B cells overexpressing miR-616 (Hep3B-miR-616 cells) showed increased ability of migration $(\mathrm{P}<0.01$, Fig. $3 \mathrm{~B})$ and invasion $(\mathrm{P}<0.05$, Fig. 3B). In contrast, miR-616 inhibitor significantly downregulated the expression level of miR-616 in MHCC97H cells $(\mathrm{P}<0.01$, Fig. $3 \mathrm{C})$, and resulted in an obviously reduced number of migrated $(\mathrm{P}<0.01$, Fig. 3D) and invaded $(\mathrm{P}<0.01$, Fig. 3D) MHCC97H cells. These results demonstrated that miR-616 can promote the invasive behavior of HCC cells.

Since the EMT process, which is regarded as a hallmark of metastasis (18), increased the migratory and invasive ability of HCC cells (19), we next investigated whether miR-616 could modulate EMT phenotype of HCC cells. Overexpression of miR-616 in Hep3B cells resulted in decreased level of E-cadherin $(\mathrm{P}<0.01$, Fig. $3 \mathrm{E})$ and increased expression of vimentin $(\mathrm{P}<0.01$, Fig. 3E). In addition, inhibition of miR-616 in MHCC97H cells led to increased level of E-cadherin $(\mathrm{P}<0.01$, Fig. 3E) and decreased expression of vimentin $(\mathrm{P}<0.01$, Fig. 3E). These results suggested that miR-616 can promote EMT of HCC cells.

PTEN is a downstream target of miR-616 in HCC cells. To clarify the underlying mechanisms by which miR-616 regulates the invasive behavior and EMT of HCC cells, we used two publicly available databases (TargetScan 6.2 and miRanda) to search potential downstream target of miR-616. PTEN, which is a well-recognized tumor suppressor and an important regulator of HCC metastasis $(20,21)$, was predicted as a downstream target of miR-616. As shown in Fig. 4A, the 3'-UTR of PTEN mRNA was found to contain the complementary sequence of miR-616. This suggests that miR-616 can potentially regulate the expression of PTEN by binding to the 3'-UTR of PTEN. To confirm this prediction, Hep3B cells transfected with miR-616 expression vector were subjected to western blotting. Overexpression of miR-616 in Hep3B cells resulted in significantly reduced expression of PTEN ( $\mathrm{P}<0.05$, Fig. 4B). It is well recognized that inhibiting PTEN expression can lead to increased phosphorylation of AKT (22). Accordingly, the phosphorylation of AKT was significantly increased after overexpressing miR-616 in Hep3B cells ( $\mathrm{P}<0.05$, Fig. 4B). Conversely, inhibiting the expression of miR-616 in MHCC97H cells resulted in significantly increased expression of PTEN ( $\mathrm{P}<0.01$, Fig. 4B) and reduced phosphorylation of AKT $(\mathrm{P}<0.01$, Fig. 4B). Next, we performed the dual-luciferase reporter assay to elucidate whether miR-616 regulated PTEN expression by directly binding to its 3'-UTR. Overexpressing miR-616 significantly inhibited the luciferase activity of PTEN with a wild-type (wt) 3'-UTR $(\mathrm{P}<0.01$, 

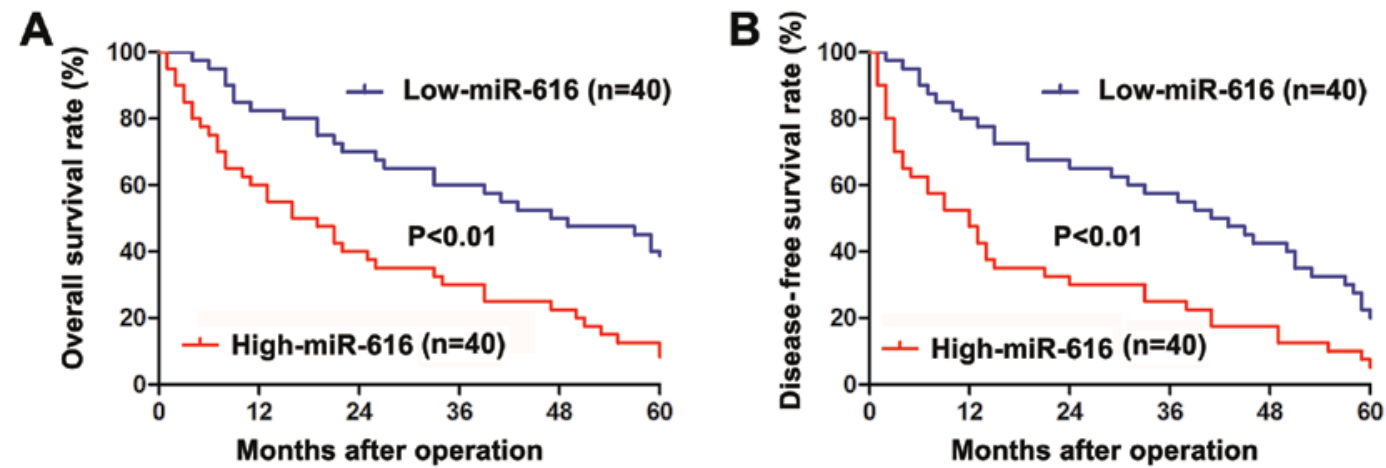

Figure 2. Prognostic value of miR-616 in HCC patients. Compared with those with low-miR-616 level, those with high-miR-616 level had significantly decreased (A) overall survival and (B) disease-free survival. The median expression level of miR-616 in $80 \mathrm{HCC}$ patients was chosen as the cut-off value. ${ }^{* *} \mathrm{P}<0.01$.

A

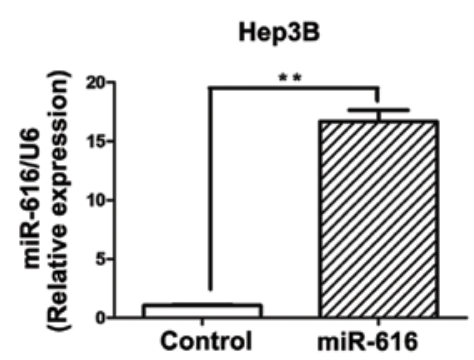

C

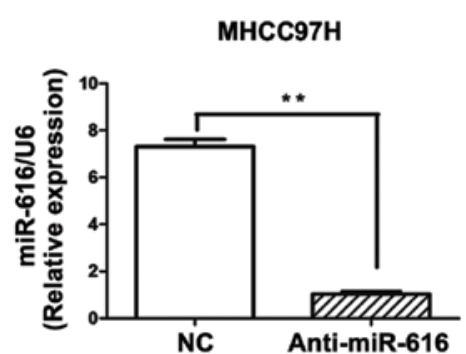

B

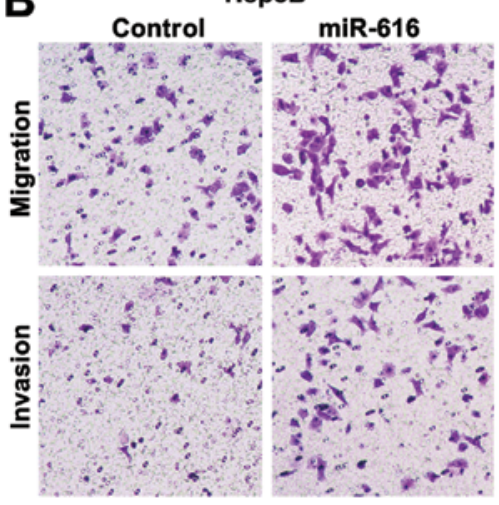

D

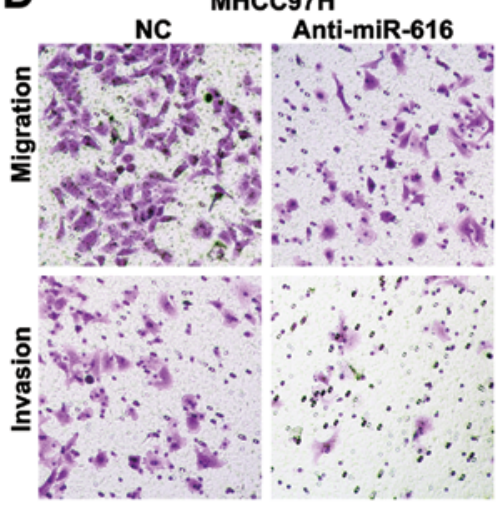

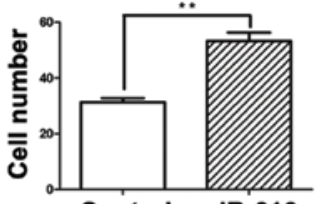

Control miR-616

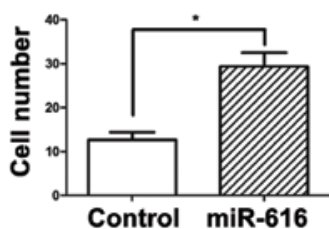

Control miR-616
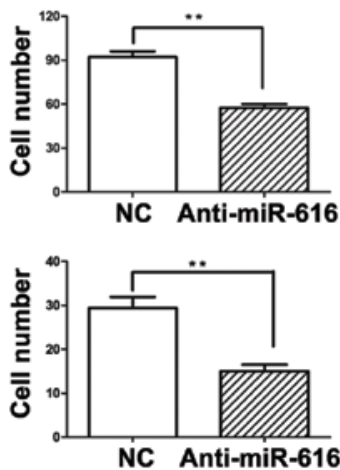

E

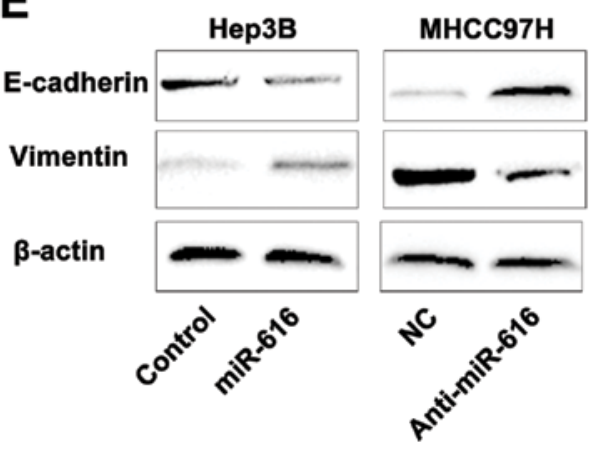

Hep3B

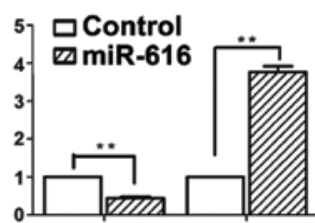

E-cadherin Vimentin
MHCC97H

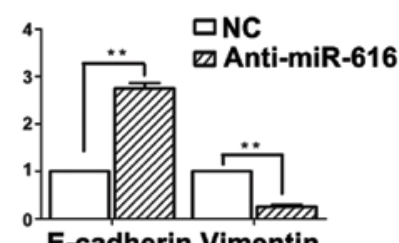

Figure 3. miR-616 promotes the migration, invasion and EMT phenotype of HCC cells. (A and B) miR-616 overexpression increased the migration and invasion of Hep3B cells. $n=3$ repeats with similar results; ${ }^{*} \mathrm{P}<0.05,{ }^{* * *} \mathrm{P}<0.01$. (C and D) Inhibiting the expression of miR-616 significantly decreased the number of migrated and invaded MHCC $97 \mathrm{H}$ cells as compared with control cells. $n=3$ repeats with similar results; ${ }^{* *} \mathrm{P}<0.01$. (E) Representative western blot analysis of E-cadherin and vimentin in Hep3B cells transfected with miR-616 mimics and MHCC $97 \mathrm{H}$ cells transfected with miR-616 inhibitor are shown. $\mathrm{n}=6$; ${ }^{* *} \mathrm{P}<0.01$. 


\section{A Mature miR-616 $33^{\prime}$ GACGAGUUUGGGAG.-..-GUUACUGA IIII IIIIIII \\ wt PTEN-3'UTR 5' ...UAAGAAACACAGCAACAAUGACU. mt PTEN-3'UTR 5' ...UAAGAAACACAGCAAGAUUCAGU.}

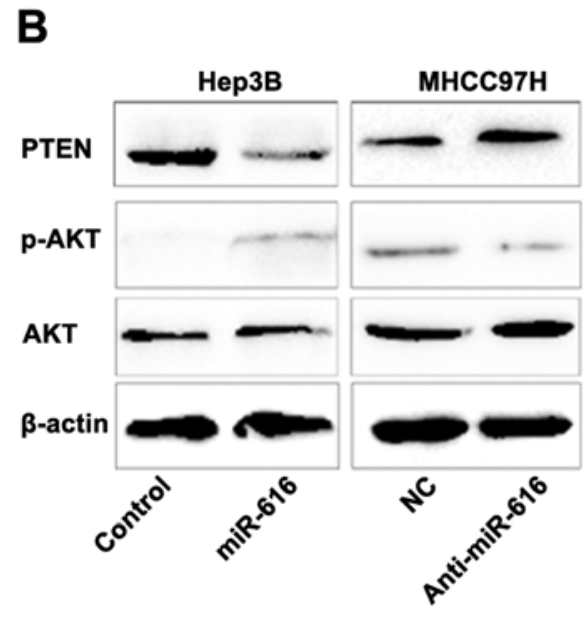

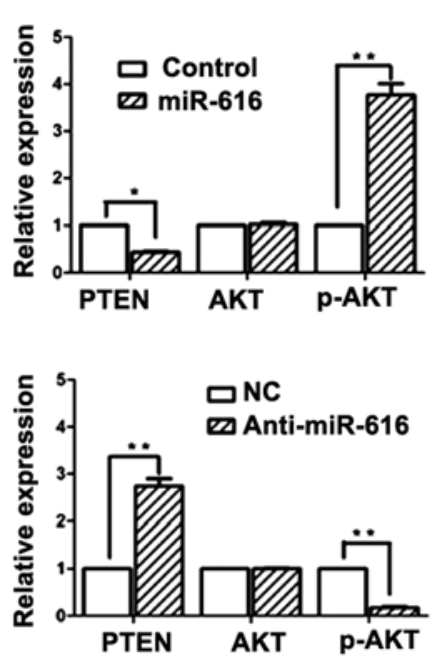

C

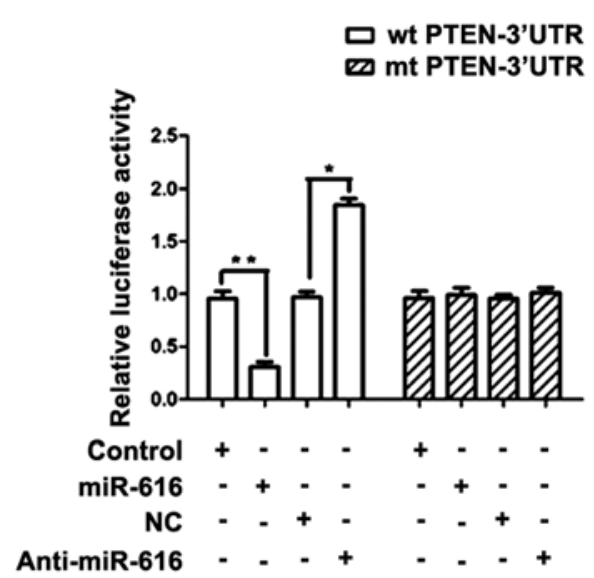

Figure 4. PTEN is identified as a direct downstream target of miR-616 in HCC. (A) miR-616 and its putative binding sequence in the 3 '-UTR of PTEN. The mutant miR-616 binding site was generated in the complementary site for the seed region of miR-616 (wt, wild-type; mt, mutant type). (B) Western blot analysis of PTEN, AKT and p-AKT expression in Hep3B cells transfected with miR-616 mimics or MHCC97H cells transfected with miR-616 inhibitors. $\mathrm{n}=6$; ${ }^{*} \mathrm{P}<0.05$, ${ }^{* *} \mathrm{P}<0.01$. (C) miR-616 overexpression significantly suppressed the luciferase activity that carried wt but not mt 3'-UTR of PTEN. Anti-miR-616 led to an obvious increase in luciferase activity of wt 3 '-UTR of PTEN. $n=3$ repeats with similar results; ${ }^{*} \mathrm{P}<0.05,{ }^{* *} \mathrm{P}<0.01$.

Fig. 4C), but had no influence on the activity of PTEN with a mutant (mt) 3'-UTR. Additionally, when anti-miR-616 was transfected, the luciferase activity of wt PTEN 3'-UTR obviously increased $(\mathrm{P}<0.01$, Fig. $4 \mathrm{C})$ while the luciferase activity of mt PTEN 3'-UTR remained unchanged.

To further validate the regulatory effect of miR-616 on PTEN expression, we compared the expression of PTEN and p-AKT in HCC tissues with low-miR-616 expression level with that of high-miR-616 expression. The IHC results showed that the PTEN expression in high-miR-616 expression group was significantly decreased when compared with that in the low-miR-616 expression group ( $\mathrm{P}<0.01$, Fig. 5A). Accordingly, the expression of p-AKT was significantly increased in highmiR-616 expression group compared to the low-miR-616 expression group $(\mathrm{P}<0.01$, Fig. $5 \mathrm{~B})$. In all, these data demonstrated that PTEN is a direct downstream target of miR-616 and miR-616 can inhibit the expression of PTEN by binding to its 3'-UTR.

miR-616 promotes EMT and metastatic ability of HCC cells through inhibiting PTEN. To further clarify the functional significance ofPTEN inmiR-616-mediatedEMT and metastatic ability of HCC cells, we transfected pCMV5-PTEN plasmids into Hep3B cells overexpressing miR-616 (Hep3B-miR-616 cells). The results of western blotting showed that transfection of pCMV5-PTEN plasmids resulted in significant increase of PTEN level in Hep3B-miR-616 cells ( $\mathrm{P}<0.01$, Fig. 6A), and led to upregulation of E-cadherin $(\mathrm{P}<0.05$, Fig. $6 \mathrm{~A})$ and reduced expression of vimentin $(\mathrm{P}<0.05$, Fig. 6A), indicating that restoring PTEN expression can reverse the EMT phenotype of Hep3B cells induced by miR-616 overexpression. Furthermore, forced expression of PTEN partly abrogated the promoting effects of miR-616 on the migration $(\mathrm{P}<0.05$, Fig. $6 \mathrm{~B})$ and invasion $(\mathrm{P}<0.05$, Fig. $6 \mathrm{~B})$ of HCC cells. These data indicated that miR-616 promotes EMT and metastatic ability of HCC cells by targeting PTEN.

\section{Discussion}

The initiation and progression of $\mathrm{HCC}$ is a complex process in which various molecules, and numerous signaling pathways are involved $(23,24)$. Emerging studies have confirmed that abnormal expression and function of miRNAs play a critical role in this process $(25,26)$. $\mathrm{miR}-616$ is a novel cancer-associated miRNA which has been found to be abnormally expressed in gastric (15), lung (16) and prostate cancer (17,27). In the present study, we initially examined the expression status of miR-616 in clinical specimens of HCC. We confirmed for the first time that compared with adjacent non-tumor tissues, the expression level of miR-616 was significantly elevated in HCC tissues. In addition, patients with recurrence and metastasis had significantly higher miR-616 levels than those without recurrence and metastasis. The clinical analysis further demonstrated that increased expression level of miR-616 was associated with adverse clinicopathological features including venous infiltration, high Edmondson-Steiner grading and advanced TNM tumor stage. Importantly, Kaplan-Meier analysis showed that increased expression of miR-616 was associated with poorer prognosis of HCC patients including overall and disease-free survival. Moreover, the expression level of miR-616 in HCC cell lines, particularly in those with high metastatic ability (HCCLM3 and MHCC97H), was significantly higher than 


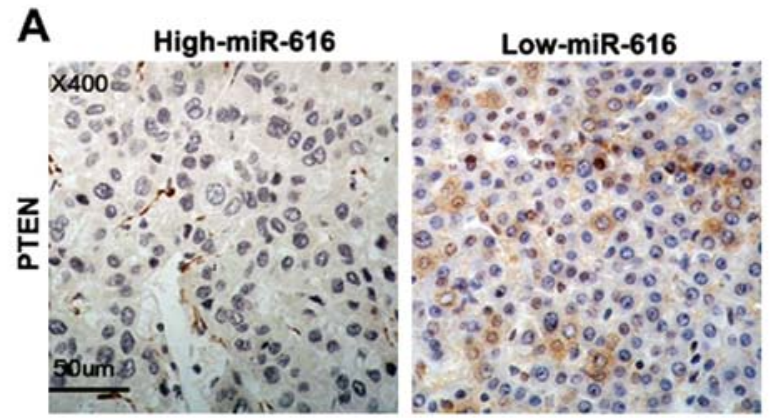

B

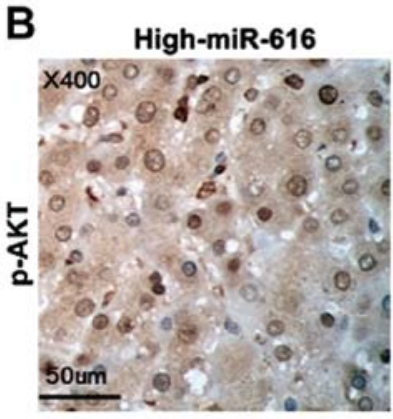

Low-miR-616

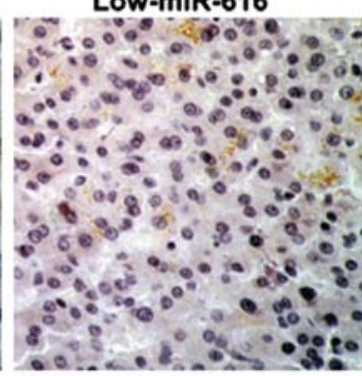

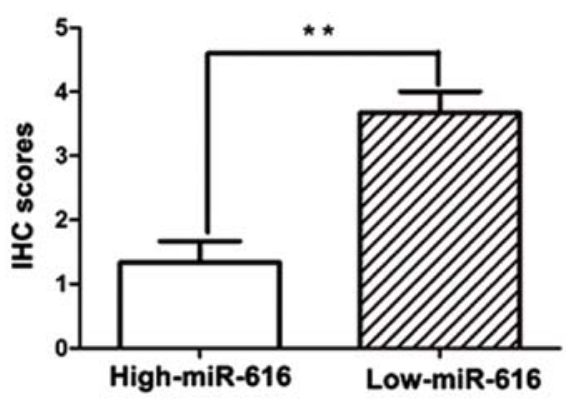

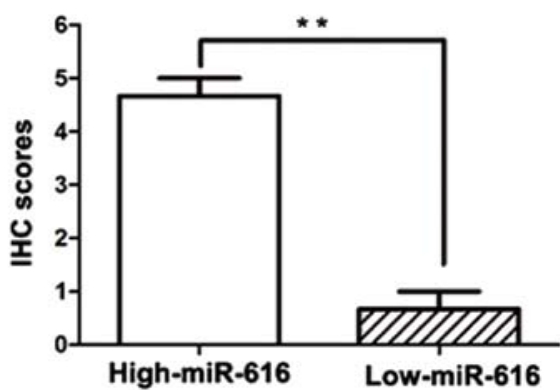

Figure 5. An inverse correlation between miR-616, PTEN and p-AKT expression is observed in HCC. (A) Representative immunostaining showed weaker staining of PTEN in miR-616-high expressing HCC tissue and higher expression of PTEN in miR-616-low expressing tissue. (B) Representative immunostaining showed increased expression of p-AKT in miR-616-high expressing HCC tissue and decreased level of p-AKT in miR-616-low expressing tissue. ${ }^{* *} \mathrm{P}<0.01$.
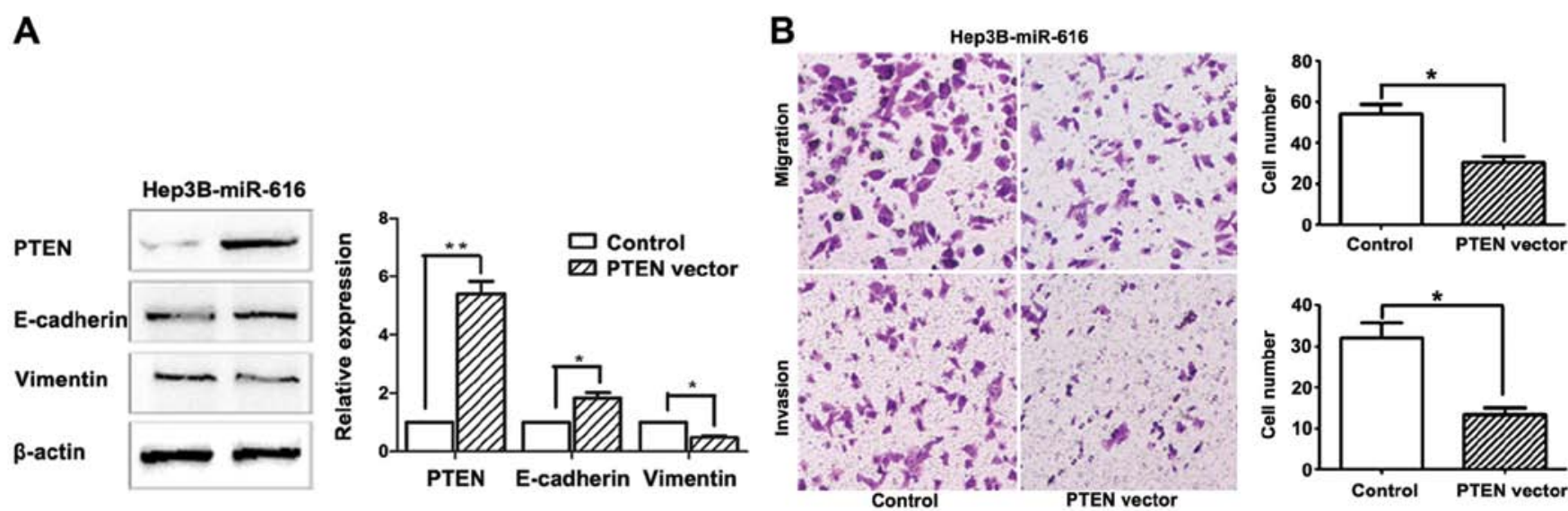

Figure 6. miR-616 induces HCC cell migration, invasion and EMT by inhibiting PTEN. (A) Western blot analysis of the expression of E-cadherin and vimentin in pCMV5-PTEN transfected miR-616 overexpressing Hep3B cells. $n=6 ;{ }^{*} \mathrm{P}<0.05,{ }^{* *} \mathrm{P}<0.01$. (B) Restoration of PTEN in miR-616 overexpressing Hep3B cells inhibited the promoting effect of miR-616 on cell migration and invasion. $\mathrm{n}=3$ repeats with similar results; ${ }^{*} \mathrm{P}<0.05$.

that in the non-transformed hepatic cell line LO2. Taken together, these results indicated that miR-616 is an important participant in the development and progression of HCC, and can serve as a prognostic marker of HCC patients.

The obvious elevation of miR-616 expression in HCC prompted us to examine the functional role of miR-616 in HCC. Since our results showed that the expression of miR-616 was associated with the recurrence and metastasis of HCC patients, we speculated that miR-616 probably influenced the migration and invasion of HCC cells. Transwell assay showed that overexpression of miR-616 promoted the migration and invasion of HCC cells while downregulation of miR-616 inhibited the metastatic behavior of HCC cells. These data suggested that miR-616 can play an active role in HCC by promoting the migration and invasion of HCC cells. EMT, a process in which epithelial cells lose cell polarity and intracellular junctions and acquire mesenchymal features $(19,28)$, can potentiate the migratory and invasive ability of HCC cells $(19,29)$. Increasing evidence has demonstrated that miRNAs, which could regulate the expression of EMT-related genes, played a critical role in the EMT process $(28,30,31)$. In the present study, we found that overexpression of miR-616 reduced the expression of E-cadherin, the epithelial marker and increased the expression of vimentin, the mesenchymal marker. Downregulation 
of miR-616 resulted in elevated expression of E-cadherin and reduced expression of vimentin. These results suggested that miR-661 promotes the EMT of HCC cells. Altogether, miR-616 may increase the invasive ability of HCC cells by inducing EMT.

Phosphatase and tensin homolog (PTEN) is a well-recognized tumor suppressor which is frequently mutated or deleted in human cancers (22). However, instead of the mutation or the deletion of PTEN, abnormal expression of PTEN was confirmed in HCC (32) and was found to promote the progression of HCC (20). However, the molecular mechanisms responsible for the abnormal expression of PTEN remains unclear. PTEN has been found to be the downstream target of several miRNAs including miR-21 $(20,33)$, miR-216a and 217 (21), miR-221 and 222 (34), miR29a (35) and miR-32 (36). In the present study, we confirmed that PTEN was a direct downstream target of miR-616 in HCC. Firstly, putative binding sequences of miR-616 were found in the 3'-UTR of PTEN after searching two publicly available databases (TargetScan and miRanda). Secondly, transfecting miR-616 mimics into Hep3B cells resulted in decreased expression of PTEN while downregulation of miR-616 in MHCC97H cells led to increased PTEN expression. The phosphorylation of Akt, which is inhibited by PTEN (37), was reduced after miR-616 overexpression and was increased after miR-616 suppression, supporting that miR-616 regulated the expression of PTEN. Additionally, the expression of PTEN in HCC tissues with high-miR-616 level was significantly lower than that in those with low-miR-616 level. This further validates the regulatory effect of miR-616 on PTEN expression. Furthermore, altering the expression level of miR-616 in Hep3B cells significantly influenced the luciferase activity of wt 3'-UTR of PTEN, while had no obvious influence on the luciferase activity of mt 3'-UTR of PTEN, suggesting that miR-616 can regulate the expression of PTEN by directly interacting with the 3'-UTR of PTEN.

Functionally, PTEN was able to influence the migration of glioma cells by modulating its downstream PI3K/Akt pathway (38). Therefore, we performed rescue experiments in Hep3B-miR-616 cells to clarify whether PTEN was involved in the promoting effect of miR-616 on the metastatic ability and EMT phenotype of HCC cells. We found that restoring the expression of PTEN abrogated the promoting effects of miR-616 on EMT and metastatic abilities of HCC cells Therefore, our results suggest that PTEN is not only a direct target of miR-616 but also a functional mediator downstream of miR-616 in HCC. Of interest is that a study of prostate cancer (17) found TFPI-2 was a direct downstream target of miR-616, and miR-616 induced androgen-independent growth of prostate cancer cells by suppressing TFPI- 2 expression. Therefore, the downstream targets and the functional significance of miRNAs in human cancers seem to be cancer-type specific.

In summary, our results demonstrated for the first time that miR-616 expression is elevated in HCC tissues, and its increased expression is associated with adverse prognostic features of HCC patients. Moreover, miR-616 is a valuable biomarker in predicting the prognosis of HCC patients. In vitro experiments demonstrated that miR-616 can promote the migration, invasion and EMT of HCC cells. Mechanistically, we found that PTEN is a downstream target of miR-616 in HCC and miR-616 exerts its functional significance on HCC cells by suppressing PTEN. Taken together, the findings from the present study indicated that miR-616 can serve as a valuable clinical biomarker, and may potentially be a promising therapeutic target in HCC.

\section{References}

1. Bosch FX, Ribes J, Díaz M and Cléries R: Primary liver cancer: Worldwide incidence and trends. Gastroenterology 127 (Suppl 1): S5-S16, 2004.

2. Karaman B, Battal B, Sari S and Verim S: Hepatocellular carcinoma review: Current treatment, and evidence-based medicine. World J Gastroenterol 20: 18059-18060, 2014.

3. El-Serag $\mathrm{HB}$ and Rudolph KL: Hepatocellular carcinoma: Epidemiology and molecular carcinogenesis. Gastroenterology 132: 2557-2576, 2007.

4. Roberts LR: Sorafenib in liver cancer - just the beginning. N Engl J Med 359: 420-422, 2008.

5. Rosa A and Brivanlou AH: MicroRNAs in early vertebrate development. Cell Cycle 8: 3513-3520, 2009.

6. Yates LA, Norbury CJ and Gilbert RJ: The long and short of microRNA. Cell 153: 516-519, 2013.

7. Calin GA and Croce CM: MicroRNA signatures in human cancers. Nat Rev Cancer 6: 857-866, 2006.

8. Chang RM, Yang H, Fang F, Xu JF and Yang LY: MicroRNA331-3p promotes proliferation and metastasis of hepatocellular carcinoma by targeting $\mathrm{PH}$ domain and leucine-rich repeat protein phosphatase. Hepatology 60: 1251-1263, 2014.

9. Budhu A, Jia HL, Forgues M, Liu CG, Goldstein D, Lam A, Zanetti KA, Ye QH, Qin LX, Croce CM, et al: Identification of metastasis-related microRNAs in hepatocellular carcinoma. Hepatology 47: 897-907, 2008.

10. Garzon R, Calin GA and Croce CM: MicroRNAs in Cancer. Annu Rev Med 60: 167-179, 2009.

11. Jansson MD and Lund AH: MicroRNA and cancer. Mol Oncol 6: 590-610, 2012.

12. Ladeiro Y, Couchy G, Balabaud C, Bioulac-Sage P, Pelletier L, Rebouissou S and Zucman-Rossi J: MicroRNA profiling in hepatocellular tumors is associated with clinical features and oncogene/tumor suppressor gene mutations. Hepatology 47: 1955-1963, 2008.

13. Giordano S and Columbano A: MicroRNAs: New tools for diagnosis, prognosis, and therapy in hepatocellular carcinoma? Hepatology 57: 840-847, 2013.

14. Cho WC: MicroRNAs: Potential biomarkers for cancer diagnosis, prognosis and targets for therapy. Int J Biochem Cell Biol 42: 1273-1281, 2010.

15. Yao Y, Suo AL, Li ZF, Liu LY, Tian T, Ni L, Zhang WG, Nan KJ, Song TS and Huang C: MicroRNA profiling of human gastric cancer. Mol Med Rep 2: 963-970, 2009.

16. Rani S, Gately K, Crown J, O'Byrne K and O'Driscoll L: Global analysis of serum microRNAs as potential biomarkers for lung adenocarcinoma. Cancer Biol Ther 14: 1104-1112, 2013.

17. Ma S, Chan YP, Kwan PS, Lee TK, Yan M, Tang KH, Ling MT, Vielkind JR, Guan XY and Chan KW: MicroRNA-616 induces androgen-independent growth of prostate cancer cells by suppressing expression of tissue factor pathway inhibitor TFPI-2. Cancer Res 71: 583-592, 2011.

18. Książkiewicz M, Markiewicz A and Zaczek AJ: Epithelialmesenchymal transition: A hallmark in metastasis formation linking circulating tumor cells and cancer stem cells. Pathobiology 79: 195-208, 2012.

19. Reichl P, Haider C, Grubinger M and Mikulits W: TGF- $\beta$ in epithelial to mesenchymal transition and metastasis of liver carcinoma. Curr Pharm Des 18: 4135-4147, 2012.

20. Meng F, Henson R, Wehbe-Janek H, Ghoshal K, Jacob ST and Patel T: MicroRNA-21 regulates expression of the PTEN tumor suppressor gene in human hepatocellular cancer. Gastroenterology 133: 647-658, 2007.

21. Xia H, Ooi LL and Hui KM: MicroRNA-216a/217-induced epithelial-mesenchymal transition targets PTEN and SMAD7 to promote drug resistance and recurrence of liver cancer. Hepatology 58: 629-641, 2013.

22. Song MS, Salmena L and Pandolfi PP: The functions and regulation of the PTEN tumour suppressor. Nat Rev Mol Cell Biol 13: 283-296, 2012. 
23. Roberts LR and Gores GJ: Hepatocellular carcinoma: Molecular pathways and new therapeutic targets. Semin Liver Dis 25: 212-225, 2005

24. Aravalli RN, Steer CJ and Cressman EN: Molecular mechanisms of hepatocellular carcinoma. Hepatology 48: 2047-2063, 2008.

25. Jiang J, Gusev Y, Aderca I, Mettler TA, Nagorney DM, Brackett DJ, Roberts LR and Schmittgen TD: Association of microRNA expression in hepatocellular carcinomas with hepatitis infection, cirrhosis, and patient survival. Clin Cancer Res 14: 419-427, 2008.

26. Murakami Y, Yasuda T, Saigo K, Urashima T, Toyoda H, Okanoue T and Shimotohno K: Comprehensive analysis of microRNA expression patterns in hepatocellular carcinoma and non-tumorous tissues. Oncogene 25: 2537-2545, 2006.

27. Haldrup C, Kosaka N, Ochiya T, Borre M, Høyer S, Orntoft TF and Sorensen KD: Profiling of circulating microRNAs for prostate cancer biomarker discovery. Drug Deliv Transl Res 4: 19-30, 2014.

28. Thiery JP and Sleeman JP: Complex networks orchestrate epithelial-mesenchymal transitions. Nat Rev Mol Cell Biol 7: 131-142, 2006.

29. Choi SS and Diehl AM: Epithelial-to-mesenchymal transitions in the liver. Hepatology 50: 2007-2013, 2009.

30. Bullock MD, Sayan AE, Packham GK and Mirnezami AH MicroRNAs: Critical regulators of epithelial to mesenchymal (EMT) and mesenchymal to epithelial transition (MET) in cancer progression. Biol Cell 104: 3-12, 2012.

31. Lee JM, Dedhar S, Kalluri R and Thompson EW: The epithelialmesenchymal transition: New insights in signaling, development, and disease. J Cell Biol 172: 973-981, 2006.
32. Wan XW, Jiang M, Cao HF, He YQ, Liu SQ, Qiu XH, Wu MC and Wang HY: The alteration of PTEN tumor suppressor expression and its association with the histopathological features of human primary hepatocellular carcinoma. J Cancer Res Clin Oncol 129: 100-106, 2003.

33. Bao L, Yan Y, Xu C, Ji W, Shen S, Xu G, Zeng Y, Sun B, Qian H, Chen L, et al: MicroRNA-21 suppresses PTEN and hSulf-1 expression and promotes hepatocellular carcinoma progression through AKT/ERK pathways. Cancer Lett 337: 226-236, 2013.

34. Garofalo M, Di Leva G, Romano G, Nuovo G, Suh SS, Ngankeu A, Taccioli C, Pichiorri F, Alder H, Secchiero P, et al: $m i R-221 \& 222$ regulate TRAIL resistance and enhance tumorigenicity through PTEN and TIMP3 downregulation. Cancer Cell 16: 498-509, 2009.

35. Kong G, Zhang J, Zhang S, Shan C, Ye L and Zhang X: Upregulated microRNA-29a by hepatitis B virus X protein enhances hepatoma cell migration by targeting PTEN in cell culture model. PLoS One 6: e19518, 2011.

36. Wu W, Yang J, Feng X, Wang H, Ye S, Yang P, Tan W, Wei G and Zhou Y: MicroRNA-32 (miR-32) regulates phosphatase and tensin homologue (PTEN) expression and promotes growth, migration, and invasion in colorectal carcinoma cells. Mol Cancer 12: 30, 2013.

37. Hers I, Vincent EE and Tavaré JM: Akt signalling in health and disease. Cell Signal 23: 1515-1527, 2011.

38. Dasari VR, Kaur K, Velpula KK, Gujrati M, Fassett D, Klopfenstein JD, Dinh DH and Rao JS: Upregulation of PTEN in glioma cells by cord blood mesenchymal stem cells inhibits migration via downregulation of the PI3K/Akt pathway. PLoS One 5: e10350, 2010 